\title{
Seeing, outer scale of optical turbulence, and coherence outer scale at different astronomical sites using instruments on meteorological balloons
}

\author{
A. Abahamid ${ }^{1}$, J. Vernin ${ }^{2}$, Z. Benkhaldoun ${ }^{1}$, A. Jabiri ${ }^{1}$, M. Azouit ${ }^{2}$, and A. Agabi ${ }^{2}$ \\ ${ }^{1}$ Laboratoire de Physique des Hautes Énergies et Astrophysique, Département de Physique, Faculté des Sciences Semlalia, \\ BP 2390, Marrakech, Maroc \\ e-mail: a.abahamid@ucam.ac.ma \\ 2 LUAN, UMR 6525, Université de Nice - Sophia Antipolis, 06108 Nice Cedex 2, France
}

Received 6 February 2004 / Accepted 24 March 2004

\begin{abstract}
Here we analyze 168 optical turbulence profiles made at nine different locations worldwide by means of free flight balloons equipped with instrumentation. Seeing $\varepsilon_{\text {FWHM }}$, optical turbulence outer scales $L_{0}$, and wavefront outer scales $£_{0}$ are derived for the different locations and taking into account the contribution of the surface layer $[0,50 \mathrm{~m}]$, the boundary layer $[0,1 \mathrm{~km}]$ and the free atmosphere $[1 \mathrm{~km}, 30 \mathrm{~km}]$. Noticeable changes are found between the different locations, mainly due to the boundary layer contribution as well as the surface layer contribution. The free atmosphere contribution seems almost the same for each site. There is a very good coherence between the outer scale derived by us and that measured using the Generalized Seeing Monitor (GSM) technique.
\end{abstract}

Key words. turbulence - atmospheric effects - balloons

\section{Introduction}

Atmospheric turbulence is responsible for the spatio-temporal fluctuations of astronomical object images obtained by means of ground-based telescopes. It is due to wavefront degradation present at the entrance of the instrument pupil plane. These degradations are more or less important depending on the atmospheric turbulence in the line of sight at the moment of the observations, and on the location.

Among the techniques to evaluate these disturbing effects, the vertical sounding of the atmosphere by means of free flight instrumented balloons is analyzed here. This survey makes it possible to have access to the thermodynamic state of different atmospheric layers from ground to $30 \mathrm{~km}$, and information on the optical turbulence of the atmosphere by the measurement of the refractive index structure constant $C_{n}^{2}$. The integrated value of this coefficient allows us to predict the atmospheric optical quality in terms of seeing $\varepsilon_{\text {FWHM }}$, as well as the local outer scale $L_{\mathrm{o}}$ that depends on the local conditions at altitude $h$ (Coulman et al. 1988; Abahamid et al. 2003). The wavefront outer scale $£_{0}$, also called the spatial coherence outer scale of the perturbed wavefront, is defined as a combination of the outer scale $L_{0}(h)$ in different atmospheric layers weighted with the refractive-index structure constant profile $C_{n}^{2}(h)$ (Lukin et al. 1958; Borgnino 1990).

An analysis of the significant data base of turbulence instrumented balloons at nine astronomical sites in the world leads to the determination of seeing $\varepsilon_{\mathrm{FWHM}}$, outer scales $L_{\mathrm{o}}$, and coherence outer scales $£_{0}$ at each location. Moreover, we determined the relative contribution of the surface layer, atmospheric boundary layer, and free atmosphere to the total disturbance. This article follows a recent survey (Abahamid et al. 2003) where all the flights were combined together.

\section{Balloon sounding}

The payload, lifted by a free flight balloon, gives us access to pressure $P$, temperature $T$, wind, humidity and the structure constant of the temperature field $C_{T}^{2}(h)$, which can be easily related to $C_{N}^{2}(h)$, assuming the hypothesis of "Kolmogorovian" turbulence, by:

$C_{N}^{2}(h)=\left(80 \times 10^{-6} \frac{P(h)}{T^{2}(h)}\right)^{2} C_{T}^{2}(h)$

where $h$ is the vertical coordinate, $P$ is the pressure expressed in $\mathrm{hPa}$ and $T$ is the absolute temperature in $\mathrm{K}$.

Our experiment involves the launching of meteorological balloons equipped with sensors which measure the microstructure of the thermal field during their free flight ascent (Vernin \& Muñoz-Tuñón 1992). They sample the atmosphere from the ground level up to $30 \mathrm{~km}$. This technique provides profiles with a good vertical resolution, typically a few meters. 
A technique similar to that of Barletti et al. (1974) is used for the temperature structure function, which is defined as:

$D_{T}(r)=\left\langle(T(x)-T(x+r))^{2}\right\rangle$,

where $r$ is the distance between two sensors, which can be chosen between a few centimeters and a few meters.

Temperature and refractive index, $T$ and $N$, are assumed to be passive and conservative additives, and thus follow the Obukhov (1949) and Yaglom (1949) spectral law. One can deduce the temperature structure constant profile $C_{T}^{2}(h)$ by:

$D_{T}(r, h)=C_{T}^{2}(h) r^{2 / 3}$.

Expression (1) allows us to link $C_{T}^{2}$ and $C_{N}^{2}$ by virtue of the known mean pressure and temperature $P$ and $T$, which are also measured on board.

$C_{N}^{2}$ (in $m^{-2 / 3}$ ) has been expressed by Tatarski (1961) as follows:

$C_{N}^{2}=a M^{2} L_{\mathrm{o}}^{4 / 3}$,

where $a$ is a constant ( $\approx 2.8$ ), $L_{\mathrm{o}}$ (in $\mathrm{m}$ ) is the outer scale of optical turbulence defined in Kolmogorov theory, and $M$ (in $\mathrm{m}^{-1}$ ), the vertical gradient of potential refractive index, is given by Coulman (1988):

$M=\frac{\delta N}{\delta h}=-\left(\frac{80 \times 10^{-6} P}{T}\right) \frac{\delta \ln \theta}{\delta h}$,

and $\theta$ is the potential temperature (in $\mathbf{K}$ ). It is defined by:

$\theta=T\left(\frac{1000}{P}\right)^{0.286}$

The seeing of full width at half the maximum is given by (Fried 1966):

$\varepsilon_{\mathrm{FWHM}}=0.98 \frac{\lambda}{r_{\mathrm{o}}}$,

when $\lambda$ is the optical wavelength and $r_{\mathrm{o}}$ is the so-called Fried parameter, which is defined as:

$r_{\mathrm{o}}=\left(16.7 \lambda^{-2} \int_{0}^{+\infty} C_{N}^{2}(h) \mathrm{d} h\right)^{-\frac{3}{5}}$,

and the expression for the seeing becomes:

$\varepsilon_{\mathrm{FWHM}}=5.25 \lambda^{-1 / 5}\left(\int_{0}^{\infty} C_{N}^{2}(h) \mathrm{d} h\right)^{\frac{3}{5}}$.

As can be appreciated from expression (9), the turbulence contribution originating from different layers can be summed linearly in terms of $C_{N}^{2}$, but may on no account be summed in terms of seeing $\varepsilon_{\mathrm{FWHM}}$, so:

$\varepsilon_{\text {total }}=\left(\sum_{i} \varepsilon_{i}^{5 / 3}\right)^{3 / 5}$, where $\varepsilon_{i}$ is the partial seeing of the $i$ th layer. Its contribution is defined as:

$\operatorname{cont}_{i}=\frac{\varepsilon_{i}^{5 / 3}}{\varepsilon_{\text {total }}^{5 / 3}}$.

The coherence outer scale $£_{0}$ of the perturbed wavefront must be distinguished from the local outer scale $L_{0}$, through the following expression:

$\mathfrak{f}_{\mathrm{o}}=\left(\frac{\int_{\mathrm{o}}^{+\infty} L_{\mathrm{o}}^{-1 / 3}(h) C_{N}^{2}(h) \mathrm{d} h}{\int_{0}^{+\infty} C_{N}^{2}(h) \mathrm{d} h}\right)^{-3}$.

\section{Statistical analysis of turbulent layers contributing to image degradation}

The turbulent energy inside the Whole Atmosphere (WA) can be divided into 2 distinct contributing elements, according to their physical origin: the Free Atmosphere (FA) above $1 \mathrm{~km}$, and the Boundary Layer (BL) which includes the first kilometer. The Surface Layer (SL) comprises the first $50 \mathrm{~m}$ of the BL. The SL is defined in this article because in most cases the balloons are not launched right at the highest point of the site but a few tens of meters below, for infrastructure convenience.

In this section we present the statistical results of the seeing $\varepsilon_{\mathrm{FWHM}}$, the optical turbulence outer scale $L_{\mathrm{O}}$ and the spatial coherence outer scale $£_{0}$ gathered for each location of the nine astronomical sites, and for each contributing layer, free atmosphere, boundary layer and surface layer using instrumented meteorological balloons.

As explained in the previous article (Abahamid et al. 2003), we processed our data within slabs of thickness $\Delta h=50 \mathrm{~m}$ to reduce the noise, which appears mainly in Eq. (5). In each $\Delta h$ slab at altitude $h_{i}$ and flight $j$ where measurements exist we compute $\left\langle C_{N}^{2}\left(h_{i}\right)\right\rangle_{j},\left\langle M\left(h_{i}\right)\right\rangle_{j}$ and $\left\langle L_{\mathrm{o}}\left(h_{i}\right)\right\rangle_{j}$ according to Eq. (4). The results obtained during twelve years of balloon sounding at nine sites are presented in Figs. 1-8.

The number and sites where the flights took place are summarized below in Table 1 .

\section{- Seeing $\varepsilon_{\mathrm{FWHM}}$ and relative atmospheric contribu- tion cont ${ }_{i}$ :}

The mean and median seeing are presented respectively in Figs. 1 and 2, for the 9 different locations. The triangles correspond to the SL contribution, squares refer to BL, Diamonds to FA and crosses represent the whole atmosphere contribution $(\mathrm{WA}=\mathrm{BL}+\mathrm{FA})$.

Figure 3 shows the seeing $\varepsilon_{\mathrm{WA}}$ inside the whole atmosphere and its variability at different locations with errors bars corresponding to \pm rms of the $\varepsilon_{\mathrm{WA}}$.

The analysis of these figures shows that the mean and median seeing values $\varepsilon_{\mathrm{FA}}$ in the free atmosphere are small and almost constant for different locations. One can estimate that the seeing in the FA is around $0.42 \pm 0.12$ arcsec. The variations in $\varepsilon_{\mathrm{SL}}, \varepsilon_{\mathrm{BL}}$ and $\varepsilon_{\mathrm{WA}}$ from one place to the other are much greater. 
Table 1. Sites, altitudes, number of balloon flights, and flight launching periods.

\begin{tabular}{|c|c|c|c|}
\hline Site & Alt. (m) & $\mathrm{Nu}$. of flights & Date \\
\hline \multirow{6}{*}{$\begin{array}{c}\text { La Palma } \\
\text { (Canaries Island) }\end{array}$} & \multirow[t]{2}{*}{2400} & \multirow[t]{2}{*}{6} & $01 / 04 / 90$ \\
\hline & & & $05 / 04 / 90$ \\
\hline & \multirow[t]{2}{*}{2200} & \multirow[t]{2}{*}{8} & $17 / 07 / 90$ \\
\hline & & & $23 / 07 / 90$ \\
\hline & \multirow[t]{2}{*}{2150} & \multirow[t]{2}{*}{7} & $01 / 11 / 95$ \\
\hline & & & $09 / 11 / 95$ \\
\hline \multirow{2}{*}{$\begin{array}{c}\text { OHP } \\
\text { (France) }\end{array}$} & \multirow[t]{2}{*}{650} & \multirow[t]{2}{*}{34} & $05 / 06 / 96$ \\
\hline & & & $24 / 07 / 02$ \\
\hline \multirow{2}{*}{$\begin{array}{c}\text { Sirene } \\
\text { (France) }\end{array}$} & \multirow[t]{2}{*}{1100} & \multirow[t]{2}{*}{19} & $17 / 07 / 02$ \\
\hline & & & $24 / 07 / 02$ \\
\hline \multirow{2}{*}{$\begin{array}{c}\text { Cerro Paranal } \\
\text { (Chile })\end{array}$} & \multirow[t]{2}{*}{2500} & \multirow[t]{2}{*}{16} & $09 / 03 / 92$ \\
\hline & & & $30 / 03 / 92$ \\
\hline \multirow[t]{2}{*}{ South Pole } & \multirow[t]{2}{*}{2835} & \multirow[t]{2}{*}{18} & $03 / 02 / 95$ \\
\hline & & & $14 / 08 / 95$ \\
\hline \multirow{2}{*}{$\begin{array}{c}\text { Cerro Pachon } \\
\text { (Chile) }\end{array}$} & \multirow[t]{2}{*}{2715} & \multirow[t]{2}{*}{45} & $13 / 01 / 98$ \\
\hline & & & $19 / 12 / 98$ \\
\hline \multicolumn{4}{|l|}{$\begin{array}{l}\text { Toulouse } \\
\text { (France) }\end{array}$} \\
\hline \multirow{2}{*}{$\begin{array}{c}\text { San Pedro Mártir } \\
\text { (Mexicco) }\end{array}$} & \multirow[t]{2}{*}{2440} & \multirow[t]{2}{*}{12} & $09 / 05 / 00$ \\
\hline & & & $21 / 05 / 00$ \\
\hline \multirow{2}{*}{$\begin{array}{l}\text { Aire sur l'Adour } \\
\text { (France) }\end{array}$} & \multirow[t]{2}{*}{57} & \multirow[t]{2}{*}{2} & $21 / 07 / 91$ \\
\hline & & & $22 / 07 / 91$ \\
\hline Total & & 168 & \\
\hline
\end{tabular}

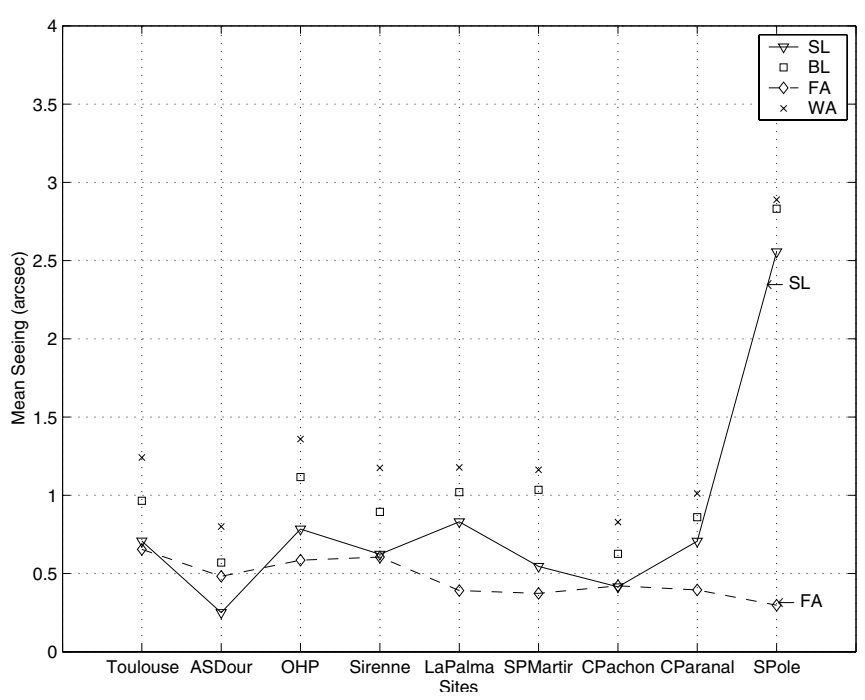

Fig. 1. Mean seeing $\varepsilon_{\mathrm{FWHM}}$ of the surface layer (SL), boundary layer (BL), free atmosphere (FA) and Whole atmosphere (WA) at the nine sites.

The contribution in $\%$ of the different slabs are presented in Figs. 4 and 5. One can see a high contribution of the boundary layer, as well as of the surface layer.

Benkhaldoun et al. (1996) used Eq. (4) to predict a $C_{N}^{2}(h)$ profile from $M(h)$ profiles deduced from meteorological sounding and assuming a universal behavior of $L_{0}(h)$ as previously given by Coulman et al. (1988). Next the predicted seeing was compared to DIMM measurements. A constant bias

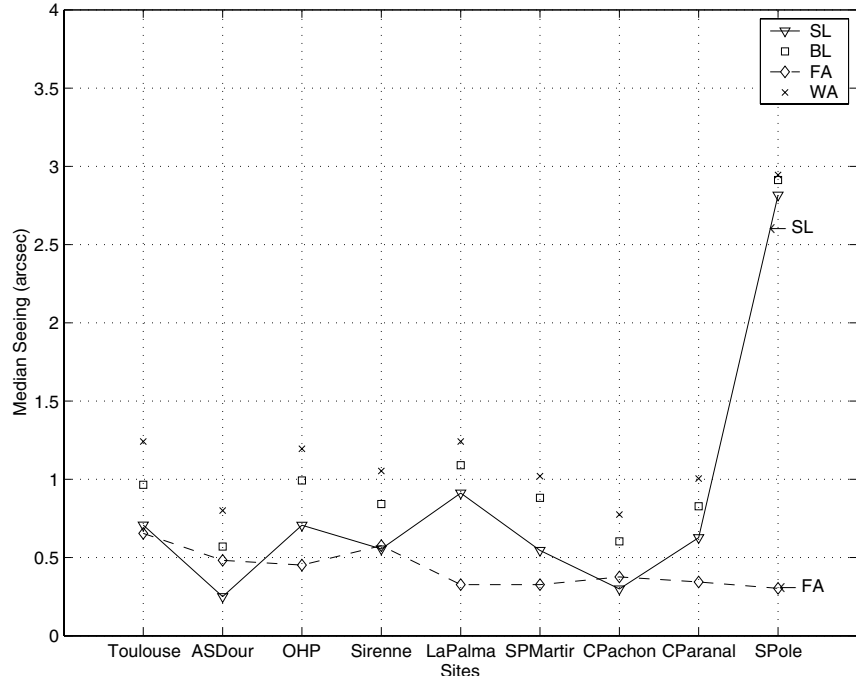

Fig. 2. Median seeing $\varepsilon_{\mathrm{FWHM}}$ of the surface layer (SL), boundary layer (BL), free atmosphere (FA) and Whole atmosphere (WA) at the nine sites.

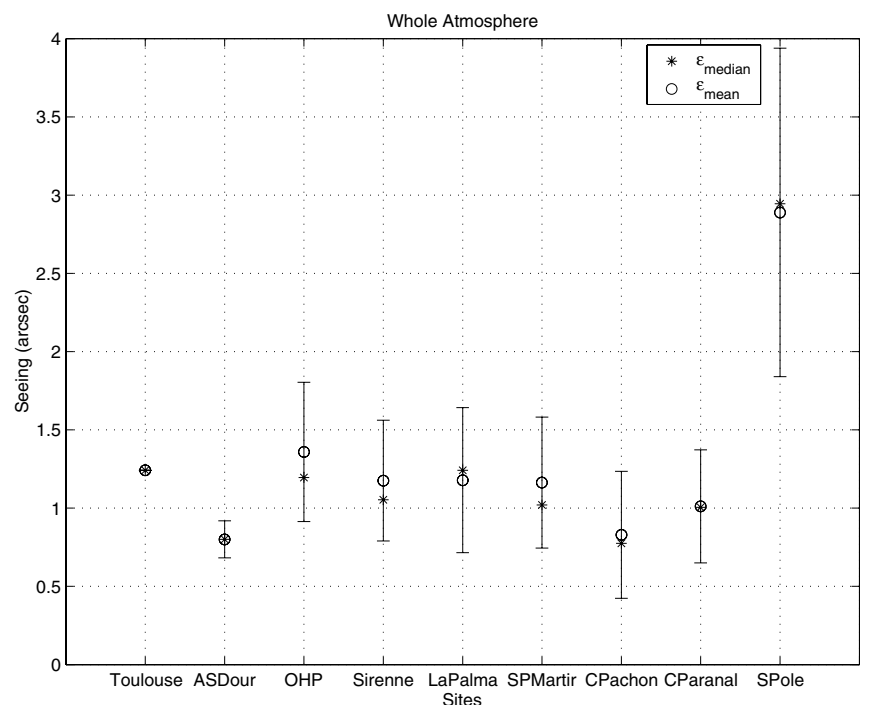

Fig. 3. Mean and median seeing $\varepsilon_{\text {FWHM }}$ calculated in the whole atmosphere at nine sites. The vertical error bars refer to the \pm rms of the $\varepsilon_{\mathrm{FWHM}}$.

of 0.3 arcsec was attributed to ground layer turbulence which has not been taken into account by the model.

Thus, we calculated the contribution to the seeing between $5 \mathrm{~m}$ and $1 \mathrm{~km}$ using the $C_{N}^{2}(h)$ model inside the boundary layer described in Abahamid et al. (2003) and repeated here, as follows:

$C_{N}^{2}(h)=1.39 \times 10^{-13} h^{-1.45}$.

The contributing seeing is estimated to 0.36 arcsec which is very close to the 0.3 arcsec bias found by Benkhaldoun et al. (1996).

\section{- Optical turbulence outer scale $L_{0}$ :}

In Figs. 6 and 7 are plotted respectively the mean and median outer scale $L_{\mathrm{o}}$ averaged over different slabs of the atmosphere. 


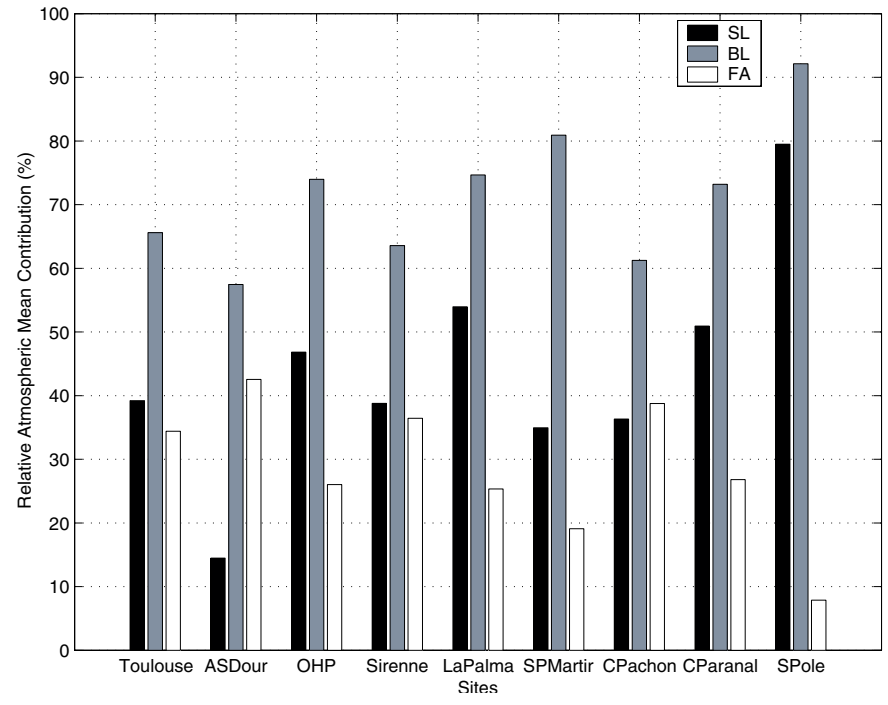

Fig. 4. Atmospheric turbulence mean contribution of the surface layer (SL), boundary layer (BL) and free atmosphere (FA) at the nine sites.

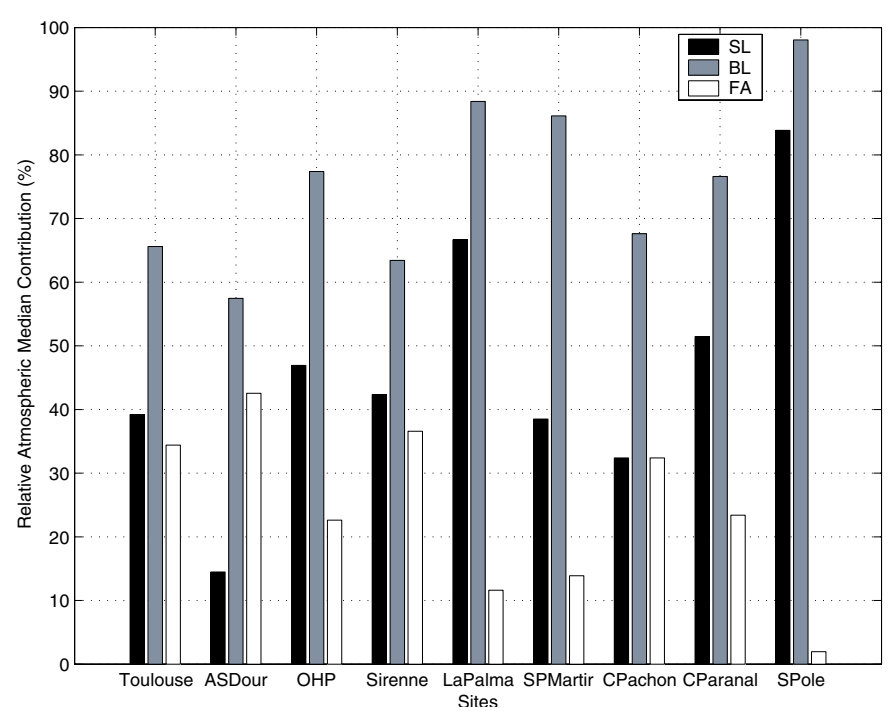

Fig. 5. Atmospheric turbulence median contribution of the surface layer (SL), boundary layer (BL) and free atmosphere (FA) at the nine sites.

Note that the variation in the values of $L_{\mathrm{o}}$ from site to site are greater in the first kilometer of the atmosphere (BL and SL) than in the free atmosphere (FA).

In the rest of the atmosphere (FA) and the whole atmosphere (WA), we registered a relatively small and stable outer scale. The mean $L_{\mathrm{o}}$ is $1.19 \pm 0.15 \mathrm{~m}$ and median $L_{\mathrm{o}}$ is $0.53 \pm$ $0.07 \mathrm{~m}$

\section{- Spatial coherence outer scale $£_{0}$ :}

Using Eq. (12), we calculated the mean $£_{\mathrm{O}_{\text {mean }}}$ and median $£_{\mathrm{o}_{\text {median }}}$ coherence outer scale. The results for each site are shown in Fig. 8.

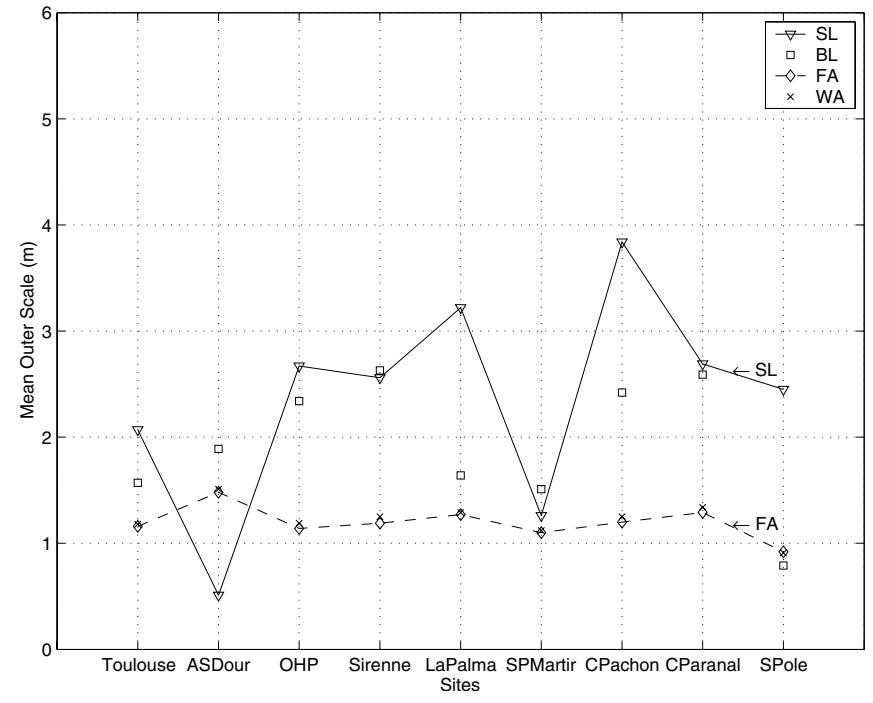

Fig. 6. Mean outer scale $L_{0}$ calculated in the surface layer (SL), boundary layer (BL), free atmosphere (FA) and Whole atmosphere (WA) at the nine sites.

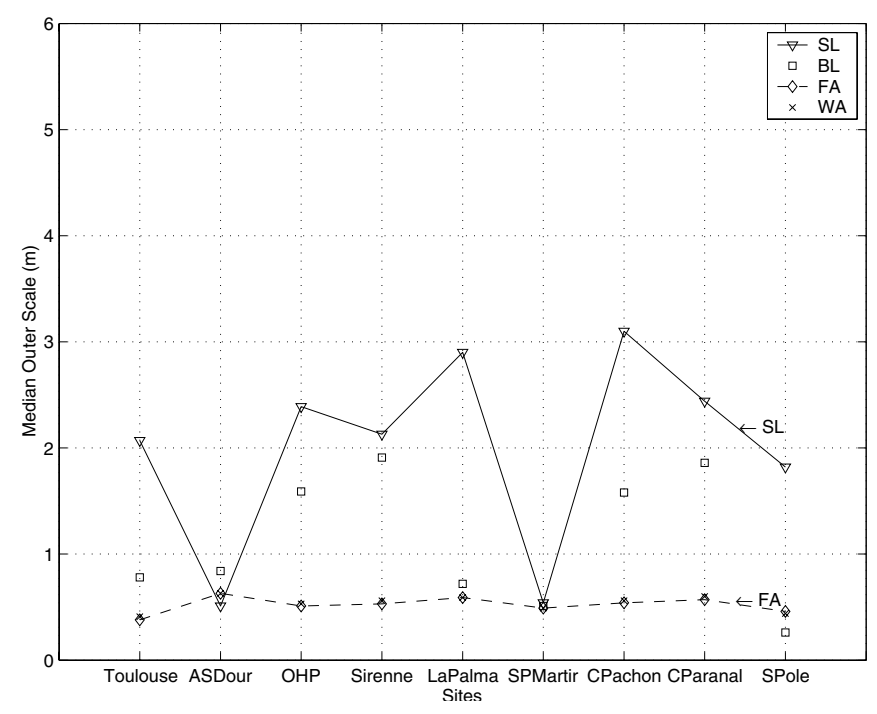

Fig. 7. Median outer scale $L_{\mathrm{o}}$ calculated in the surface layer (SL), boundary layer (BL), free atmosphere (FA) and Whole atmosphere (WA) at the nine sites.

\section{Discussion and conclusion}

The results presented here have been derived from 168 free-flight balloons at nine astronomical sites. Based on this data set, we provide not only the statistical properties at each location, but also for each slab of the atmosphere. Seeing $\varepsilon_{\text {FWHM }}$, optical turbulence outer scale $L_{0}$, and coherence outer scale $£_{0}$ are analyzed for the different locations and atmospheric slabs.

Noticeable changes of the seeing $\varepsilon_{\text {FWHM }}$ are found at the various sites, mainly due to the boundary layer and the surface layer. This might be due to a major influence of orographic effects on the mean flow, as also noticed by Erasmus (1986).

The free atmosphere has a much lower and constant contribution of about $0.42 \pm 0.12$ arcsec comparable to the 0.46 arcsec obtained at Mauna Kea (Hawaï, 


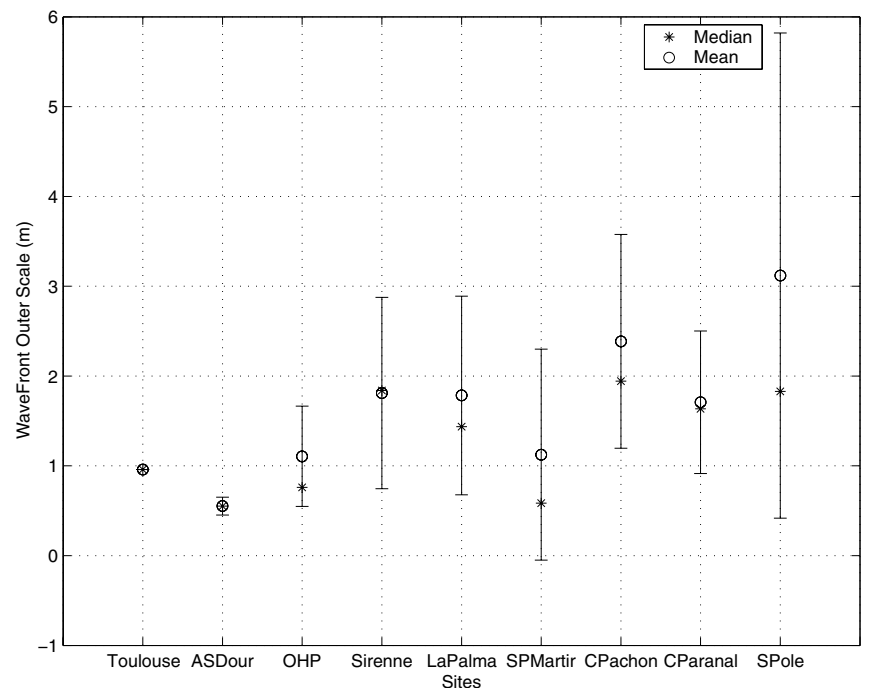

Fig. 8. Mean and median coherence outer scale $£_{0}$ calculated in the whole atmosphere at the nine sites. The vertical error bars refer to the \pm rms of the $\mathfrak{f}_{0}$.

Table 2. Whole atmosphere mean seeing at different locations as measured by GSM and our balloon data.

\begin{tabular}{ccc}
\hline \hline Site & $\begin{array}{c}\text { GSM seeing } \\
(\operatorname{arcsec})\end{array}$ & $\begin{array}{c}\text { Balloon seeing } \\
(\operatorname{arcsec})\end{array}$ \\
\hline $\begin{array}{c}\text { La Palma } \\
\text { (Canaries Island) }\end{array}$ & 1.14 & $1.18 \pm 0.46$ \\
\hline $\begin{array}{c}\text { Cerro Pachon } \\
\text { (Chile) }\end{array}$ & 0.89 & $0.83 \pm 0.41$ \\
\hline $\begin{array}{c}\text { Cerro Paranal } \\
\text { (Chile) }\end{array}$ & 0.91 & $1.01 \pm 0.36$ \\
\hline $\begin{array}{c}\text { San Pedro Mártir } \\
\text { (Mexicco) }\end{array}$ & 1.15 & $1.16 \pm 0.42$ \\
\hline
\end{tabular}

Roddier et al. 1990), the 0.34 arcsec obtained at La Silla (Chile, Sarazin 1987), and the 0.4 arcsec measured by the SCIDAR at La Palma (Canaries Island, Vernin et al. 1992).

In the whole atmosphere, the seeing $\varepsilon_{\mathrm{WA}}$ determined by means of balloon sounding at La Palma, Cerro Pachon, Cerro Paranal, and San Pedro Mártir are agree very well with GSM results measured by Ziad et al. (2000) and Ziad (2003) summarized below, in Table 2 .

The value of the spatial coherence outer scale $£_{0}$ is controversial because the available measurements provide scattered values ranging from a few meters to kilometers (Avila et al. 1997), likely due to the fact that $£_{0}$ is model dependent. The outer scale values provided by the GSM are derived from the von Kármán model of the phase spectrum (Ziad et al. 2000). The use of another model would change the outer scale vales $£_{0}$. In Table 3 we compare our $£_{0}$ results with the $£_{0}$ deduced from GSM following a simple adjustment:

$£_{\text {bal }}=0.076 £_{\mathrm{gsm}}$

which has been deduced by Abahamid et al. (2003), during the Cerro Pachon campaign, in October 1998, the only campaign where GSM and balloon were used together.
Table 3. Mean coherence outer scale $£_{0}$ at different location as measured by GSM and our balloon data.

\begin{tabular}{ccc}
\hline \hline Site & $£_{\text {gsm }}(\mathrm{m})$ & $\mathfrak{f}_{\text {bal }}(\mathrm{m})$ \\
\hline La Palma & 11.9 & $23.47 \pm 14.55$ \\
(Canaries Island) & & \\
\hline & & October 1998 (7 flights) \\
Cerro Pachon & 28.4 & $29 \pm 13.22$ \\
(Chile) & & Whole campaign \\
& 28.4 & $22.40 \pm 15.66$ \\
\hline $\begin{array}{c}\text { Cerro Paranal } \\
\text { (Chile) }\end{array}$ & 23.9 & 10.43 \\
\hline $\begin{array}{c}\text { San Pedro Mártir } \\
\text { (Mexicco) }\end{array}$ & 26.9 & \\
\hline
\end{tabular}

One can notice a large scatter between GSM and balloonderived $£_{0}$ which might be explained by the non simultaneity of the observations and by the fact that $£_{\mathrm{o}}$ is known to change very rapidly (Avila et al. 1997).

Acknowledgements. We are very indebted to many people from OHP, Sirene, ORM, SPM, CTIO, ESO and South Pole astronomical sites who helped us in the organization of the infrastructure in order to launch our balloons. We thank very much the agencies who financed the launching campaings, such as US Air Force (contract F61775-02-C002), IAC, UNAM, AURA, ESO and NSF. This study was made possible also thanks to Agence Universitaire de la Francophonie which gave a grant to one of the authors, A.A.

\section{References}

Abahamid, A., Jabiri, A., Vernin, J., et al. 2004, A\&A, 416, 1193

Avila, R., Ziad, A., Borgnino, J., et al. 1997, J. Opt. Soc. Am. A, 14, 3070

Barletti, R., Ceppatelli, G., Moroder, E., Paterno, L., \& Righini, A. 1974, J. Geophys. Res., 79, 4545

Benkhaldoun, Z., Jabiri, A., \& Vernin, J. 1996, Ap\&SS, 239, 237

Borgnino, J. 1990, Appl. Opt., 29, 1863

Coulman, C. E., Vernin, J., Coqueugniot, Y., \& Caccia, J. L. 1988, Appl. Opt., 27, 155

Erasmus, A. 1986, Int. Conf. on telescope sites, Flagstaff

ESO-VLT working group 1987, in ESO VLT Report No. 55, ed. M. Sarazin, Garching

Fried, D. L. 1966, J. Opt. Soc. Am., 56, 1372

Lukin, V., Nosov, E., \& Fortes, B. 1958, Opt. Atmosf., 10, 162

Obukhov, A. 1949, Izv. Akad. Nauk. S.S.S.R, Ser. Geograf. Geofiz., 13,58

Roddier, F., Cowie, L., Graves, J. E., et al. 1990, in Advanced Technology Optical Telescopes IV, ed. L. D. Barr (Tucson), 485

Tatarski, V. I. 1961, Wave Propagation in a Turbulent Medium (New York: Dover)

Vernin, J., \& Muñoz-Tuñón, C. 1992, A\&A, 257, 811

Yaglom, A. M. 1949, Dan. S.S.S.R, 69, 743

Ziad, A., Conan, R., Tokovinin, A., Martin, F., \& Borgnino, J. 2000, Appl. Opt., 39, 5415

Ziad, A. 2003, Habilitation à diriger des recherches, LUAN, UMR 6525, Université de Nice - Sophia Antipolis, 06108 Nice Cedex 2, France 